5. Bosch J, Mastai R, Kravetz D, et al. Effects of propranolol on azygous venous blood flow and hepatic and systemic haemodynamics in cirrhosis. Hepatology 1984; 4:1200-1205.

6. Bosch J, Mastai R, Kravetz D, et al. Measurement of azygous blood flow in the evaluation of portal hypertension in patients with cirrhosis. J Hepatol 1985; 1:125-129.

7. Lebrec D, DeFleury P, Rueff B, et al. Portal hypertension size of esophageal varices, and risk of gastrointestinal bleeding in alcoholic cirrhosis. Gastroenterology 1980; 79:1139-1144.

8. Lebrec D, Poynard T, Bernuau J, et al. A randomized controlled study of propranolol for prevention of recurrent gastrointestinal bleeding in patients with cirrhosis: a final report. Hepatology 1984; 4:355-358.

9. Valla D, Bercoff E, Manu Y, et al. Discrepancy between wedged hepatic venous pressure and portal venous pressure after acute propranolol administration in patients with alcoholic cirrhosis. Gastroenterology 1984; 86:1400-1403.

10. Burroughs AK, Jenkins WJ, Sherlock S, et al. Controlled trial of propranolol for the prevention of recurrent variceal haemorrhage in patients with cirrhosis. N Engl J Med 1983; 309:1539-1542.

11. Zelen $M$. The analysis of several $2 \times 2$ contingency tables. Biometrika $1971 ; 58: 129-137$.

12. Conn HO. Propranolol in portal hypertension: problems in paradise? Hepatology $1984 ; 4: 560-564$.

\section{COSTS OF LIVER TRANSPLANTATION: PRIMUM NON OBFUSCARE}

Van Thiel DH, Tarter R, Gavaler JS, Potanko WM and Schade $R R$. Liver transplantation in adults. An analysis of costs and benefits at the University of Pittsburgh. Gastroenterology 1986; 90:211-216.

\section{EDITOR'S ABSTRACT}

The costs and benefits of liver transplantation in adult patients at the University of Pittsburgh were reviewed for the period from 1981 through 1984. Indirect costs such as those to support the surgical and hepatology programs, the operating rooms and the clinical pathology department were ignored, and only those costs generated by the liver transplant program were considered in this analysis. Benefits to the patients are survival itself. Over $8.5 \%$ of those who leave the hospital return to full-time employment or other useful activity, including normal, albeit complicated, pregnancies. Most deaths occur during the first 3 months, after which there is a slow decline during the first year. Costs to the patient and/or the third party payers are enormous, and include evaluation as a transplant candidate, procurement of the donor liver, the transplantation itself and lifelong medical-surgical follow-up and immunosuppression plus the management of the undesirable consequences of the whole procedure. Laboratory tests alone average over $\$ 52,000$ per patient. The consumption of blood and blood products is great and averages 30 units per patient. Duration of hospital admissions averaged about 55 days per patient, of which about 10 were in intensive care units. Total dollar costs were not calculated, however. The benefits to medicine are considerable in the acquisition of new knowledge and skills appli- cable to other disorders and other patients. The authors conclude that with further experience, the costs will decrease and the benefits will increase.

\section{COMMENTS}

The recent paper by Van Thiel et al. first promises to be quite interesting and important. In the introduction, they note: "The following is a cost-benefit analysis of liver transplantation as applied to a single institution...." Unfortunately, it is not a cost-benefit analysis, nor does it add significantly to our understanding of the costs associated with liver transplantation.

The key element of cost-benefit analysis is that both the inputs and outputs are expressed in monetary terms (1). The application of cost-benefit analysis to medical problems requires the assignment of a dollar figure to a human life; this can be done, but only with obvious criticism (2). An alternate approach is cost-effectiveness analysis in which a cost is assigned to the health care process; but rather than assigning a dollar figure to a life, the life itself is used as the unit of measurement. Thus, cost-effectiveness analysis presents information such as the cost of colon cancer screening to detect one cancer of the colon, or to avert one cancer-related death. Additionally, both cost-benefit analysis and cost-effectiveness analysis use the patient as the unit of analysis. It is important to define both of these methods of analysis for several reasons: (i) they are the standard techniques employed by health services researchers; (ii) the definition of cost-benefit analysis is quite exact; (iii) precise definitions permit assessment of the nature and significance of analysis of the costs of medical care.

Despite their introduction, Van Thiel et al. performed neither a cost-benefit nor a cost-effectiveness analysis. They first describe the "costs" and "benefits" to a transplantation center. They note that because dollar values are "difficult to compare between institutions, the costs, whenever possible, will be reported in terms of number of personnel, days, or some other unit of service." The costs to the institution are listed as the additional institutional costs for such personnel as a senior surgeon, anesthesiologists, secretaries (etc.). The "benefits" to the institution (and participating physicians) include national and international recognition, local publicity, the ability to attract faculty and the like. The authors then switch their orientation to the patient and attempt to identify costs and benefits. Patient-related "costs" include such items as time spent in the ICU, units of blood, the costs of laboratory tests, preoperative hospital costs and posthospital hospital costs, where "costs" are expressed in terms of charges. Aside from the obvious inconsistency in assigning personnel "costs" to the hospital and laboratory "costs" to the patient, the authors confuse the identification of costs in general.

Despite the profession's proclamation that liver transplantation is no longer an experimental technology, society appears to be incompletely convinced (3-5; Evans, R. W., Lancet 1983; 2:1306, Correspondence). The key question asked by society is: "Do the benefits justify the costs?" We live at a time of increasing concern about the costs of medical care. Liver transplantation is both high 
cost and life saving for a small number of patients. It serves, therefore, as a bellwether condition. While physicians have made great advances in the care of such patients, fewer advances have been made in our ability to arrive at the true costs of that care. Without knowing the true costs of care, society is concerned that the few patients who do benefit from liver transplantation siphon off a disproportionate amount of resources from other patients also in need of health care (6).

Hospital "costs" can be expressed by a number of terms: accounting costs, economic costs and charges (7). The usual measure chosen when "costs" are discussed is to utilize hospital charges as a "cost" measure as illustrated by Van Thiel et al. Charges are the prices set by a hospital for a small subset of private-paying patients and commercial third party payors. The charges have little, if anything to do with a hospital's "costs." The charge is a price set by the hospital in response to the market, it is usually well above "costs," but in some patients it may actually be below "costs."

The distinction between economic costs and accounting costs is critical when one evaluates technologies like liver transplantation. As noted by Finkler: "Economic cost will be used to refer to the price paid by the health care institution for the resources it consumes" (7). It is the economic cost that is important when one evaluates a new technology. It is essential to distinguish clearly this economic cost from the hospital's accounting cost. Accounting cost refers to the "costs" attributed to patients via the hospital's accounting system.

Hospital accounting systems accumulate cost information at the departmental level, and these accounting costs are then attributed to a patient on the basis of some statistic (e.g., length of stay). The attribution problem is, in fact, compounded because before costs can be attributed to a patient, all of a hospital's costs must be attributed first to hospital activities that directly serve patients and, therefore, generate income for the institution. Thus, costs from nonincome-producing departments such as administration, maintenance, dietary and the like must be attributed first to patient care centers and then, ultimately, to a patient. This process of cost attribution to the income-producing departments is accomplished via an accounting technique called a multiple step-down, in which the first nonrevenue-producing center's costs (e.g., administration) is "distributed" to all the remaining centers (8). This process is continued until all of the nonrevenue-producing centers' costs are distributed to the revenue-producing centers. The resulting "costs" in the revenue producing center's are then allocated to patients. This final allocation to the patient represents the hospital's accounting costs.

Two points are crucial concerning accounting costs: first, the "costs" that end up in the final revenue-producing cost centers are largely a function of the order of the accounting step-down. 'Thus, if one began the stepdown first with maintenance costs rather than administrative costs, the accounting costs in the laboratory (a revenue-producing cost center) would differ. Second, the final allocation from revenue-producing cost centers to patients is based upon average measures (e.g., length of stay, time in the operating room). From this brief de- scription of the accounting costs of a hospital, one can see that they differ from both charges and true economic costs. Indeed, they are neither.

In looking at a program like liver transplantation, it is clear that the only appropriate measure is the economic costs of the program. In establishing a new program, it is important to assess the additional prices paid by the health care institution for the resources that the new program consumes (7). From the discussion above, charges can be dismissed out of hand, as they have nothing at all to do with the economic costs of a hospital. The accounting costs could be useful at an individual institution, but only if competing treatment modalities use similar final revenue-producing centers so that the bias due to the step-down procedure is minimized. It is clear that this condition is not met with liver transplantation. If one could make the case that similar revenue centers are used, one must then evaluate the effect of the final attribution of costs to patients within each final revenue center. If there is a bias in this allocation, then all of the final accounting costs will either over- or underestimate the economic costs.

In reviewing studies that have attempted to evaluate the costs of liver transplantation, we see that there is a systematic bias that tends to underestimate the accounting costs attributed to liver transplant patients. As discussed above, costs are allocated from final revenueproducing centers to patients on the basis of some average statistic. Thus, for example, patients are allocated a percentage of the total ICU costs as a function of their length of ICU stay, and surgical patients are allocated a percentage of the operating room costs on the basis of the time spent in the operating room, etc. Such allocation statistics, in effect, assign an average cost to each patient. This method works well if all patients in an ICU use similar amounts of resources and thus have similar economic costs. As patients in the same final revenueproducing center are more heterogeneous, the use of an allocation statistic based on averages will systematically understate the economic costs of very resource-intensive cases, and overstate the costs in low resource cases. Wagner et al. (9) have recently identified this type of cross-subsidization in an ICU and demonstrated that actual resource use differed by a factor of two in cases that were allocated the same accounting costs.

Why is this detail important when considering liver transplantation? In a recent paper, Luebs (10) outlined some of the cost considerations in liver transplantation programs. By ignoring the charge information and focusing on the actual resources consumed, important information is provided. Luebs notes that, because of their liver transplant program, operating room staffing was increased, more high acuity nursing care was needed and the time necessary to clean a transplant isolation room was four times as long as for a normal isolation room. Therefore, when one looks at the allocation of accounting costs from any one of the final cost centers to liver transplant patients, the accounting costs systematically underestimate the economic costs of the program.

Is it important to know what liver transplantation costs society? I think so, although others would question this assertion. Krom and Gips note that "liver transplan- 
tation generates so many questions related to pathophysiology of the liver and transplantation, that results of related research and its effect on health care are spinoffs which cannot be estimated economically" (11). Furthermore, as Van Thiel et al. noted, benefits to the institution (and presumably the physician) include national and international recognition. While each of the above is true, they clearly identify an inherent conflict of interest when those directly engaged in a line of research are asked to assess its worth. In juxtaposition to these assertions are those who would argue on economic grounds that liver transplantation is not justified. Of course, it costs less not to intervene with expensive medical treatments than to allow the disease to bring about death in its course. But a decision to withhold available, effective intervention, based solely on economic consideration, is repugnant to most physicians as well as to most of the public. What is the answer?

An ongoing public debate about the use of sophisticated and costly medical technology such as liver transplantation is needed. Informed groups of citizens, with input from health care professionals, are probably best able to render such judgments free of the conflicts of interest inherent among the health professionals (5). Clearly needed is credible economic data about liver transplantation, which, as noted above, will be difficult to obtain given the complexity of hospital accounting. However, choosing to ignore this complexity may obfuscate the understanding and impair the resolution of this important public issue.
Laurence F. McMahon, JR. M.D., M.P.H. Assistant Professor Department of Internal Medicine University of Michigan

Ann Arbor, Michigan 48109-0376

\section{REFERENCES}

1. Weinstein MC, Stason WB. Foundations of cost-effectiveness analysis for health and medical practices. N Engl J Med 1977; 296:716721.

2. Avorn J. Benefit and cost analysis in geriatric care. Turning age discrimination into health policy. N Engl J Med 1984; 310:12941301.

3. National Institutes of Health Consensus Conference Development Statement. Liver Transplantation June 20-23, 1983. Hepatology 1984; 4:13-45.

4. Liver transplantation 1985 and beyond. Lancet $1985 ; 1: 27-28$.

5. Annas GJ. Regulating the introduction of heart and liver transplantation. Am J Public Health 1985; 75:93-95.

6. Evans RW. Health care technology and the inevitability of resource allocation and rationing decisions. JAMA 1983; 249:2047-2053, 2208-2219.

7. Finkler JA. The distinction between cost and charges. Ann Intern Med 1982; 96:102-109.

8. Berman HJ, Weeks LE. Cost analysis. In: The Financial Management of Hospitals, Bureau of Hospital Administration, School of Public Health, the University of Michigan, 1971, Ann Arbor, Michigan.

9. Wagner DP, Wineland TD, Knaus WA. The hidden costs of treating severely ill patients: charges and resource consumption in an intensive care unit. Health Care Financing Rev 1983; 5:81-86.

10. Luebs HW. Cost considerations. Sem Liver Dis 1985; 5:402-411.

11. Krom RAF, Gips CH. Skills and resources needed for liver transplantation. Hepatology 1984; 4:72S-75S. 